\title{
A COMPARISON OF THE DIAGNOSTIC VALUE OF MAGNETIC RESONANCE MAMMOGRAPHY VERSUS ULTRASOUND MAMMOGRAPHY IN MODERATE- AND HIGH-RISK BREAST CANCER PATIENTS
}

\author{
Sara Yahyazadeh', Rahele Mehraeen ${ }^{2}$
}

1MD, Student Research Committee, School of Medicine, Babol University of Medical Sciences, Babol, Iran.

${ }^{2}$ Assistant Professor, Department of Paediatric Radiology, Babol University of Medical Sciences, Babol, Iran.

\begin{abstract}
BACKGROUND
ABSTRACT

Due to the high prevalence of breast cancer, it is important to use screening methods for the diagnosis of early stages and faster treatment of this disease; better diagnosis should improve survival and quality of life for patients. Mammography, which is the preferred screening method for 40- to 70-year-old women, currently has limitations for the diagnosis of specific lesions. That is why screening with ultrasonography and magnetic resonance imaging (MRI) have recently received more attention. This study determined the sensitivity and specificity of these imaging methods in one hospital.
\end{abstract}

\section{MATERIALS AND METHODS}

This diagnostic descriptive study was conducted on women referred to surgical and gynaecological clinics of Rouhani Teaching Hospital in Babol, Northern Iran, who had a moderate or high risk of breast cancer. After MRI, the patients underwent ultrasonography and 6-month follow-up, with biopsies where needed. The researchers analysed the results to determine the diagnostic value of MRI and breast ultrasound, and to compare them.

\section{RESULTS}

A total of 106 women with an average age of $43.93 \pm 11.51$ years were included. Out of 57 people who had Breast ImagingReporting and Data System (BIRADS) 3 by the ultrasound, 44 (77\%) people had BIRADS 3 on follow-up, but 13 people were in other groups. Of 59 people who had BIRADS 3 in the MRI, 54 (91\%) had BIRADS 3 after the follow-up too. The sensitivity, specificity, positive and negative predictive values (PPV and NPV), and accuracy of ultrasound were 91.7\%, 87.2\%, 47.8\%, 98.8\% and $87.7 \%$, respectively. These amounts were 100\%, 98.9\%, 92.3\%, 100\% and $99.1 \%$ for MRI, respectively. The difference for specificity, PPV and accuracy between ultrasound and MRI were significant ( $p=0.006, p=0.007, p=0.001$, respectively), but not significant for sensitivity and NPV.

\section{CONCLUSION}

MRI has higher sensitivity, specificity and accuracy and less false positive and negative results for the early diagnosis of breast cancer in comparison with ultrasound.

\section{KEY WORDS}

Breast Neoplasms, Diagnosis, Magnetic Resonance Imaging, Ultrasonography.

HOW TO CITE THIS ARTICLE: Yahyazadeh S, Mehraeen R. A comparison of the diagnostic value of magnetic resonance mammography versus ultrasound mammography in moderate and high-risk breast cancer patients. J. Evolution Med. Dent. Sci. 2018;7(53):5629-5633, DOI: 10.14260/jemds/2018/1245

\section{BACKGROUND}

Cancer is a major health problem that affects the health of every community.(1) Cancer is the second most common cause of death after cardiovascular diseases in developed countries, and is the third cause of death after cardiovascular diseases and disasters in less developed countries.(2) Among the various types of cancer, breast cancer is the most common, which accounts for $23 \%$ of all cancers in women. It is the most lethal malignancy among women and is one of the most important health concerns of women in the world.(3-5)

'Financial or Other Competing Interest': We would like to thank the Vice Chancellor for the Research of Babol University of Medical Sciences for Financial Support.

Submission 17-11-2018, Peer Review 14-12-2018,

Acceptance 20-12-2018, Published 31-12-2018.

Corresponding Author:

Dr. Rahele Mehraeen,

Department of Paediatric Radiology,

Babol University of Medical Sciences,

Ganjafrooz Street, Babol, Mazandaran, Iran.

E-mail: rahelemehraeen4@gmail.com

DOI: $10.14260 /$ jemds $/ 2018 / 1245$
According to statistics from the Cancer Research Center in Iran, every year about 8, 500 new breast cancer cases are registered in the country, and 1, 400 people die from this cancer.(6) Also, there are currently about 40,000 people living with the disease in the country.(6)

The World Health Organization and the American Cancer Society state that the best way to control breast cancer is early diagnosis. $(7,8)$ Given that $50 \%$ of breast cancer patients in Iran only refer at an advanced stage of the disease,(6) the need for early diagnosis of the disease is important.

For early detection of breast cancer in asymptomatic patients, American Cancer Society recommends to use the screening methods, including breast self-examination, clinical breast examination, mammography, ultrasonography, and magnetic resonance imaging (MRI).(9) Ultrasonography and mammography are not only helpful in screening for breast cancer, but also for grading benign versus malignant status of observed lesions.(10,11) The complete evaluation of a mass found on mammography and appearing to be malignant requires several steps. Left and right breast should be compared in each view. The masses that have convex edges become denser, due to aggregation and collapse the normal structure of the breast. Real masses are seen in different 
views and can even be seen with local pressure. The margin, density, location and size characteristics of a mass should be evaluated. The presence of cluster pleomorphic microcalcification, with or without a soft tissue mass, is the mammography primary sign of breast cancer. Forms of benign calcification are also recognized. Mammography also has limitations in the detection of some lesions. Lobular carcinoma in situ does not have a definite appearance on mammography, when clinically obscured, they are often found in the vicinity of unspecified calcification, but benign masses are also found by mammography.(12) Furthermore, mammography has limitations in a dense breast; when there is an increase in the fibroglandular tissue ratio to fat, and the mass is hidden in mammography due to the high tissue density.(13)

Most cases of diagnostic ultrasound are performed in a targeted way for investigating abnormal mammographic or palpable lumps. Ultrasound can prevent the need for biopsy by identifying normal or definitely benign lesions. Ultrasound is also a guide for a range of interventions. Some of the features that increase the risk of malignancy revealed by ultrasound include a mostly solid, hypoechoic, taller than wide, thick echogenic margin, irregular margin, angulated, acoustic shadowing, and microcalcification nodules.(14) The percentage of diagnosis by ultrasound of interval cancers in patients previously screened by mammography is $3 \%$, and suggests that ultrasound is very useful as a secondary screening for patients having dense breast in mammography. However, breast ultrasonography is operator-dependent and is also limited in the diagnosis of isoechoic and non-mass-like lesions. Furthermore, ultrasound is not able to fully assess deeper tissues in those people with large breasts.

The common uses of magnetic resonance imaging currently include women with hereditary breast cancer risk, known as BRCA mutation, for the assessment of response to neoadjuvant chemotherapy, and preoperative staging in recently-diagnosed cancers. Breast magnetic resonance imaging is performed at magnet of 1.5 Tesla or above, and is performed in three planes T1, T2, fat suppression, 3D before contrast, and two or three images after 6-8 minutes of contrast injection. Each lesion should be examined in terms of shape, margin, internal structure, signal characteristics and enhancing characteristics.(15) As mentioned, the diagnosis of invasive lobular carcinoma is very difficult by ultrasound, mammography, and clinical examination - hence, magnetic resonance imaging is the preferred diagnostic method for this type of cancer.(16) It can also be used to investigate suspicious lesions in mammography and ultrasound, especially in highand moderate-risk patients, and to prevent unnecessary biopsy or to identify malignant lesions. However, magnetic resonance mammography is costly and time consuming, and its use is therefore limited.

This study investigated the diagnostic accuracy of magnetic resonance imaging compared with the other methods of imaging in moderate- and high-risk patients, with the aim of early diagnosis of breast cancer in these patients.

\section{MATERIALS AND METHODS}

This diagnostic descriptive study was performed on 106 women, who were referred to surgical and gynaecological clinics of Rouhani Teaching Hospital in Babol, Northern Iran.
The inclusion criteria included a first-degree cancer history, ovarian cancer history in the patient, proven breast cancer history in the patient, proven BRCA gene in the patient, Breast Imaging-Reporting and Data System(17) (BIRADS) 3 or more in mammography, as well as patients who did not have the ability to full assessment by mammography due to high breast tissue density. All of these people were at moderate- and high-risk for breast cancer. They were recommended to undergo ultrasound and magnetic resonance imaging mammography. The exclusion criteria included the lack of referral for ultrasound and/or magnetic resonance imaging.

All patients provided written consent, and the patient's demographic information, including age, family history of breast and ovarian cancer, history of chest irradiation, oral contraceptive consumption, and mass detected by physician were recorded in a predesigned checklist.

Ultrasonography was performed by Samsung WS80 device for women's ultrasound, and the information was noted about every mass: solid or cystic mass, size, marginal characteristics, echogenicity, presence or absence of microcalcification and vascular pattern. The location of the mass was reported in the ultrasonography based on the O'clock position and distance from the nipple. Magnetic resonance imaging was performed by a General Electric device in a 1.5 Tesla magnet, which examined the size, marginal characteristics, shape, lesion signal, and post-gadolinium injection enhancement in T1, T2, and fat suppression images.

Patients with suspicious lesions requiring biopsy were placed in BIRADS 4-5 and underwent core needle biopsy. The cytology response was classified as benign, malignant, suspected to be malignant and unidentifiable. Biopsy was performed using a 14-gauge needle under sterile conditions with local anesthesia at the Radiology Center of Rouhani Hospital, and samples were sent for pathology examination. All suspect lesions were biopsied when there were multiple lesions.

If the patient wished to be investigated in another center, the results were tracked and recorded.

In the case of BIRADS 3, they returned for about 6-month follow-up with targeted ultrasound or one-way mammography, and in the case of BIRADS 1-2, they returned for routine screening. If the BIRADS 4-5 was `in the follow-up examination, biopsy was taken from the lesion and the results were compared with pathology, which is the gold diagnostic standard for lesions with BIRADS 4-5, and patients were referred for treatment. In each patient, a higher BIRADS was considered as the criterion for diagnostic or therapeutic action.

The obtained data were entered in SPSS v22 software and analyzed. The data underwent the descriptive analysis to provide the number, percentage, and mean \pm standard deviation (SD). The chi-square test was used to compare the sensitivity, specificity, positive and negative predictive values (PPV and NPV), and accuracy between ultrasound and MR mammography. The significance level was less than 0.05 in all tests.

The ethical committee of Babol University of Medical Sciences approved the present research. We collected informed consent from all subjects. The patients' information was kept confidential. 


\section{RESULTS}

A total of 106 women with an average age of $43.9 \pm 11.5$ (range, 25-70) years were included in this study. Among the patients participating in this study, 58 patients had undergone mammography before entering in the study, and all patients had undergone ultrasound and MRI. Results showed that $21.7 \%$ of the patients had a history of cancer, $9.4 \%$ had a history of cancer in close relatives, $17.9 \%$ of the patients had palpable masses, $27 \%$ had dense fibroglandular tissue in mammography and $23 \%$ had big and fatty tissue on ultrasonography.

Table 1 indicates the results of ultrasound and MRI for BIRADS 3. Out of 57 people who had BIRADS 3 in the ultrasound, 44 people still had BIRADS 3 on follow-up as well, but 13 people were assigned to other groups - that is, $77 \%$ of the patients were found to be the same in both the first examination and follow-up. Of 59 people who had BIRADS 3 in the MRI, 54 were assessed as BIRADS 3 after the follow-up - that is, the results of $91 \%$ of patients in the BIRADS 3 group were the same in the first examination and follow-up

Tables 2 and 3 show the diagnostic values of ultrasound and MRI for BIRADS 4-5. As found, the sensitivity, specificity, PPV, NPV and accuracy of ultrasound were 91.7\%, 87.2\%, $47.8 \%, 98.8 \%$ and $87.7 \%$, respectively. These amounts were $100 \%, 98.9 \%, 92.3 \%, 100 \%$ and $99.1 \%$ for MRI, respectively. The difference for specificity, PPV and accuracy between ultrasound and MRI was significant, but not for sensitivity and NPV.

\begin{tabular}{|c|c|c|c|c|}
\hline & \multicolumn{2}{|c|}{ Follow-Up } \\
\hline & & & + & - \\
\hline \multirow{2}{*}{ Ultrasound } & \multirow{2}{*}{ BIRADS 3} & + & 44 & 13 \\
\hline & & - & 15 & 34 \\
\hline \multirow{2}{*}{ MRI } & \multirow{2}{*}{ BIRADS 3} & + & 54 & 5 \\
\hline & & - & 5 & 42 \\
\hline
\end{tabular}

Table 1. Comparison of the results for Breast ImagingReporting and Data System (BIRADS) 3 obtained in the

first examination and after six-month follow-up between magnetic resonance imaging (MRI) and ultrasound mammography

\begin{tabular}{|c|c|c|c|c|}
\hline & \multicolumn{2}{|c|}{ Biopsy } \\
\hline & & & + & - \\
\hline \multirow{2}{*}{ Ultrasound } & \multirow{2}{*}{ BIRADS 4-5 } & + & 11 & 12 \\
\hline & & - & 1 & 82 \\
\hline \multirow{2}{*}{ MRI } & \multirow{2}{*}{ BIRADS 4-5 } & + & 12 & 1 \\
\hline & & - & 0 & 93 \\
\hline \multicolumn{5}{|c|}{\begin{tabular}{|} 
Table 2. Comparison of the results of biopsy for Breast \\
Imaging-Reporting and Data System (BIRADS) 4-5 \\
obtained in the first examination and after six-month \\
follow-up between magnetic resonance imaging (MRI) and \\
ultrasound mammography
\end{tabular}} \\
\hline
\end{tabular}

\begin{tabular}{|c|c|c|c|c|c|}
\hline Methods & Sensitivit & Specificity & PPV & NPV & Accuracy \\
\hline Ultrasound & $91.7 \%$ & $87.2 \%$ & $47.8 \%$ & $98.8 \%$ & $87.7 \%$ \\
\hline MRI & $100 \%$ & $98.9 \%$ & $92.3 \%$ & 100 & $99.1 \%$ \\
\hline P-value & 0.307 & 0.006 & 0.007 & 0.288 & 0.001 \\
\hline \multicolumn{6}{|c|}{$\begin{array}{l}\text { Table 3. The diagnostic value of magnetic resonance } \\
\text { laging (MRI) and ultrasound mammography for Breast } \\
\text { Imaging-Reporting and Data System (BIRADS) 4-5 }\end{array}$} \\
\hline
\end{tabular}

\section{DISCUSSION}

In this cross-sectional study that compared the diagnostic values of ultrasound imaging and breast MRI in patients referred for breast cancer examination in a Northern Iranian hospital, MRI has been shown to have greater sensitivity and specificity for the early diagnosis of breast cancer.

In general, it can be said that the diagnosis of breast cancer or its exclusion are the main objectives of breast imaging. Mammography is known as an effective screening test for the early diagnosis of breast cancer. The sensitivity of mammography in dense and heterogeneous breasts can be reduced to $30-48 \% .^{(18-20)}$ The combination of the reduced sensitivity of mammography and the increased prevalence of cancer in the dense breast has led to a trend toward additional screening with ultrasonography or MRI imaging.(21) Further investigation is needed to determine the sensitivity and specificity of MRI and ultrasound in the early diagnosis of breast cancer.

The most important objective in this study was to estimate the diagnostic value of MRI imaging versus breast ultrasound imaging in patients with breast cancer. The sensitivity of MRI in this study was estimated to be $100 \%$ for the BIRADS 4-5 group, and the ultrasound sensitivity was $92 \%$ for these patients. In the study by Kuhl et al.(22) The sensitivity of the MRI imaging method was close to that of the present study (91\%), and the researcher showed that the sensitivity of this method is significantly higher than that of mammography or ultrasonography. A study by Berg et al.(18) Showed a sensitivity of $95 \%$ for MRI and $94 \%$ for ultrasonography in breast cancer diagnosis in suspected breast cancer patients, which MRI had a less sensitivity but ultrasound had a higher sensitivity compared with that of our study. The sensitivity of MRI in the review by Mann et al.(23) Was also $96 \%$.

Other studies conducted on this subject have estimated the sensitivity of MRI imaging in the diagnosis of cancerous lesions to be lower than our study. The sensitivity was reduced to $81 \%$ in the study by Sardanelli et al.(24) $79.5 \%$ in the study by Kriege et al.(25) and $77 \%$ in the study by Warner et al.(26) However, it has been shown in all the three studies that MRI has more sensitivity than other diagnostic methods in breast cancer, and can have a better association with the results of the gold standard, i.e. pathology, in diagnosis of the lesions. In contrast to the overall result of these studies, Berg et al. (27) showed that in patients with high risk of breast cancer, the addition of ultrasound or MRI screening to mammography not only fails to result in a better diagnosis, but also increases false positive findings. It should be noted that in the study by Berg et al.(27) Despite the sensitivity of $100 \%$, the specificity for this combinational screening method was estimated to be $65 \%$, which could be a valid justification for this claim. In the present study, the specificity of MRI and ultrasound imaging were calculated to be $99 \%$ and $88 \%$, respectively, for BIRADS 4-5. As seen in our study, MRI imaging had a high degree of specificity in addition to high sensitivity. In a study by Li et al.(28) and Warner et al.(26) the specificity for MRI was estimated to be $95 \%$, and to be $89 \%$ in the study by Keriege et al.(25)

The results of MRI in the present study for BIRADS 4-5 group showed both higher sensitivity and also specificity in comparison with ultrasonography. On this basis we can say that in the BIRADS 4-5 group, MRI was a more valuable 
diagnostic method than ultrasonography in diagnosing suspected lesions. However, Li et al.(28) concluded that MRI has the best results in the group of patients with BIRADS 4 compared with other methods, but would not have better results than other methods in BIRADS 5. Similarly, Strobel et al.(29) Also reported the best results for MRI in the BIRADS 4 group.

In this study, most importantly, false positive cases in MRI in BIRADS 4-5 were estimated to be 1 out of $13(7.6 \%)$, comparable to ultrasound with 11 of 23 lesions with $52 \%$ false positive cases. In the study by Strobel et al.(29) Which also evaluated and compared ultrasound with MRI, it was shown similarly that in BIRADS 4, MRI was able to prevent unnecessary biopsy in $92 \%$ of cases and the false negative rate for MRI was calculated to be $0 \%$. In a study by Yeh et al.(30) It was shown that MRI may estimate the residual disease to be more than $(6 \%)$ or less than $(23 \%)$ of the actual level.

The analysis of the data in this study showed that MRI is an effective imaging method to accurately evaluate lesions in suspected cases. Based on the enhancement pattern, it is able to prevent some patient from unnecessary biopsy, which is a stress-inducing process for most of the patients. In a study by Houssami et al.(31) It was shown that the average additional diagnosis for MRI was estimated to be $16 \%$. Newer data suggests that MRI may not reduce the rate of surgical recurrence and results in a false positive increase in terms of diagnosis and unnecessary surgeries,(31) but this conclusion contrasts with the findings of the present study. In general, it can be argued that there is currently little quality evidence to support the routine use of pre-surgical MRI to choose the preferred therapeutic model.

\section{CONCLUSION}

It has been shown that in Northern Iran, MRI has higher sensitivity, specificity and accuracy and less false positive and negative results for the early diagnosis of breast cancer in comparison with ultrasound. For further studies, it is suggested that the patients be categorized into different groups based on their risk factors, in order to determine which method has the greatest diagnostic value. Future studies should also involve a longer period of observation.

\section{ACKNOWLEDGMENT}

We would like to thank the Vice Chancellor for the Research of Babol University of Medical Sciences for financial support. We are also thankful to Dr. Hossein Shahabandaz, Dr. Soraya Khafri and Dr. Mohammad Ranaei for their help during the study.

\section{REFERENCES}

[1] Siegel RL, Miller KD, Jemal A. Cancer Statistics, 2017. CA Cancer J Clin 2017;66(1):7-30.

[2] Sabatino SA, Lawrence B, Elder R, et al. Effectiveness of interventions to increase screening for breast, cervical, and colorectal cancers: nine updated systematic reviews for the guide to community preventive services. Am J Prev Med 2012;43(1):97118.

[3] Nafissi N, Saghafinia M, Motamedi MH, et al. A survey of breast cancer knowledge and attitude in Iranian women. J Cancer Res Ther 2012;8(1):46-9.
[4] Banegas MP, Bird Y, Moraros J, et al. Breast cancer knowledge, attitudes and early detection practices in United States-Mexico border Latinas. J Women's Health (Larchmt) 2012;21(1):101-7.

[5] Porter P. "Westernizing" women's risks? Breast cancer in lower-income countries. $N$ Engl $J$ Med 2008;358(3):213-6.

[6] Tahergorabi Z, Moodi M, Mesbahzadeh B. Breast cancer: a preventable disease. Journal of Birjand University of Medical Sciences 2014;21(2):126-41.

[7] Fentiman IS. Fixed and modifiable risk factors for breast cancer. Int J Clin Pract 2001;55(8):527-30.

[8] Moodi M, Rezaeian M, Mostafavi F, et al. The study of mammography screening behavior based on stage of change model in Isfahanian women of age 40 and older: a population-based study. ZUMS 2013;21(84):24-35.

[9] Benson JR, Jatoi I, Keisch M, et al. Early breast cancer. Lancet 2009;373(9673):1463-79.

[10] Chakkrapani R, Durairaj B. Diagnostic accuracy of mammography and ultrasonography for breast diseases. J Evolution Med Dent Sci 2018;7(5):592-6.

[11] Adarsh A, Krishna KR, Kodumur V, et al. Role of breast ultrasound in evaluation of Birads 3 and Birads 4 breast masses. J Evolution Med Dent Sci 2017;6(45):3524-8.

[12] Łuczyńska E, Heinze-Paluchowska S, Hendrick E, et al. Comparison between breast MRI and contrastenhanced spectral mammography. Med Sci Monit 2015;21:1358-67.

[13] Nothacker M, Duda V, Hahn M, et al. Early detection of breast cancer: benefits and risks of supplemental breast ultrasound in asymptomatic women with mammographically dense breast tissue. A systematic review. BMC Cancer 2009;9:335.

[14] Corsetti V, Houssami N, Ghirardi M, et al. Evidence of the effect of adjunct ultrasound screening in women with mammography-negative dense breasts: interval breast cancers at 1 year follow-up. Eur J Cancer 2011;47(7):1021-6.

[15] Heywang-Köbrunner SH, Hacker A, Sedlacek S. Magnetic resonance imaging: the evolution of breast imaging. Breast 2013;22(Suppl 2):S77-S82.

[16] Bazzocchi M, Facecchia I, Zuiani C, et al. Diagnostic imaging of lobular carcinoma of the breast: mammographic, ultrasonographic and MR findings. Radiol Med 2000;100(6):436-43.

[17] Berg WA, Campassi C, Langenberg P, et al. Breast imaging reporting and data system: inter-and intraobserver variability in feature analysis and final assessment. AJR Am J Roentgenol 2000;174(6):176977.

[18] Berg WA, Gutierrez L, NessAiver MS, et al. Diagnostic accuracy of mammography, clinical examination, US, and MR imaging in preoperative assessment of breast cancer. Radiology 2004;233(3):830-49.

[19] Nagao Y, Kawaguchi Y, Sugiyama Y, et al. Relationship between mammographic density and the risk of breast cancer in Japanese women: a case-control study. Breast Cancer 2003;10(3):228-33. 
[20] Ursin G, Ma H, Wu AH, et al. Mammographic density and breast cancer in three ethnic groups. Cancer Epidemiol Biomarkers Prev 2003;12(4):332-8.

[21] Berg WA. Rationale for a trial of screening breast ultrasound: American College of Radiology Imaging Network (ACRIN) 6666. AJR Am J Roentgenol 2003;180(5):1225-8.

[22] Kuhl CK, Schrading S, Leutner CC, et al. Mammography, breast ultrasound, and magnetic resonance imaging for surveillance of women at high familial risk for breast cancer. J Clin Oncol 2005;23(33):8469-76.

[23] Mann RM, Hoogeveen YL, Blickman JG, et al. MRI compared to conventional diagnostic work-up in the detection and evaluation of invasive lobular carcinoma of the breast: a review of existing literature. Breast Cancer Res Treat 2008;107(1):1-14.

[24] Sardanelli F, Giuseppetti GM, Panizza P, et al. Sensitivity of MRI versus mammography for detecting foci of multifocal, multicentric breast cancer in fatty and dense breasts using the whole-breast pathologic examination as a gold standard. AJR Am J Roentgenol 2004;183(4):1149-57.

[25] Kriege M, Brekelmans CT, Boetes C, et al. Efficacy of MRI and mammography for breast - cancer screening in women with a familial or genetic predisposition. $\mathrm{N}$ Engl J Med 2004;351(5):427-37.

[26] Warner E, Plewes DB, Hill KA, et al. Surveillance of BRCA1 and BRCA2 mutation carriers with magnetic resonance imaging, ultrasound, mammography, and clinical breast examination. JAMA 2004;292(11):131725.
[27] Berg WA, Zhang Z, Lehrer D, et al. Detection of breast cancer with addition of annual screening ultrasound or a single screening MRI to mammography in women with elevated breast cancer risk. JAMA 2012;307(13):1394-404.

[28] Li E, Li J, Song Y, et al. A comparative study of the diagnostic value of contrast-enhanced breast MR imaging and mammography on patients with BI-RADS 3-5 microcalcifications. PLoS One 2014;9(11):e111217.

[29] Strobel K, Schrading S, Hansen NL, et al. Assessment of BI-RADS category 4 lesions detected with screening mammography and screening US: utility of MR imaging. Radiology 2015;274(2):343-51.

[30] Yeh E, Slanetz P, Kopans DB, et al. Prospective comparison of mammography, sonography and MRI in patients undergoing neoadjuvant chemotherapy for palpable breast cancer. AJR Am J Roentgenol 2005;184(3):868-77.

[31] Houssami N, Hayes DF. Review of preoperative magnetic resonance imaging (MRI) in breast cancer: should MRI be performed on all women with newly diagnosed, early stage breast cancer? CA Cancer J Clin 2009;59(5):290-302. 